\title{
FIXED POINTS OF ORIENTATION REVERSING HOMEOMORPHISMS OF THE PLANE
}

\author{
KRYSTYNA KUPERBERG
}

(Communicated by Frederick R. Cohen)

\begin{abstract}
Let $h$ be an orientation reversing homeomorphism of the plane onto itself. If $X$ is a plane continuum invariant under $h$, then $h$ has a fixed point in $X$. Furthermore, if at least one of the bounded complementary domains of $X$ is invariant under $h$, then $h$ has at least two fixed points in $X$.
\end{abstract}

\section{INTRODUCTION}

In 1951, M. L. Cartwright and J. C. Littlewood (see [6]) proved that if $X$ is a nonseparating, connected, compact, nonempty subset of the plane, invariant under an orientation preserving homeomorphism $h$ of the plane, then $h$ has a fixed point in $X$. Simpler proofs of the Cartwright-Littlewood theorem were later provided by O. H. Hamilton in [7] and by Morton Brown in [5]. In [2], H. Bell proved the Cartwright-Littlewood theorem for an arbitrary homeomorphism of the plane (see also [1] and [4]). The results of this paper depend strongly on Bell's theorem for orientation reversing homeomorphisms of the plane.

Throughout this paper, $h$ is an orientation reversing homeomorphism of the plane $R^{2}$ onto itself, and $X$ is a continuum in the plane (i.e. a nonempty, connected and compact subset of $R^{2}$ ), invariant under $h$. The components of $R^{2}-X$ are called the complementary domains of $X$.

M. Barge asked whether $h$ has always a fixed point in $X$, and in some cases, for instance if $X$ has exactly two complementary domains, whether $h$ has two fixed points in $X$. The following theorem provides an answer to Barge's question:

Theorem. If at least one of the bounded complementary domains of $X$ is invariant under $h$, then $h$ has at least two fixed points in $X$. Otherwise, $h$ has at least one fixed point in $X$.

Received by the editors August 15, 1989.

1980 Mathematics Subject Classification (1985 Revision). Primary 55M20; Secondary 54F15, $54 \mathrm{H} 25,58 \mathrm{C} 30$.

Key words and phrases. Fixed point. 
An easy example of two tangent circles with disjoint interiors and an orientation reversing homeomorphism of $R^{2}$ interchanging the circles shows that if no bounded complementary domain of a separating plane continuum $X$ is invariant, then $h$ may have only one fixed point in $X$.

The author would like to thank Morton Brown for his valuable comments.

\section{Preliminary Lemmas}

Let $A$ be an annulus in $R^{2}$ and let $X$ be a separating plane continuum contained in $A$ such that the two boundary components of $A, C_{1}$ and $C_{2}$, are in different complementary domains of $X$ (equivalently, $X$ separates the annulus $A$ between its boundary components $C_{1}$ and $C_{2}$ ). Let $H$ be an orientation reversing homeomorphism of $R^{2}$ onto itself. Assume that both $A$ and $X$ are invariant under $H$.

Let $\widetilde{A}$ be the universal covering space of $A$, and let $p: \widetilde{A} \rightarrow A$ be the covering map. A homeomorphism $\widetilde{H}: \widetilde{A} \rightarrow \widetilde{A}$ such that the diagram

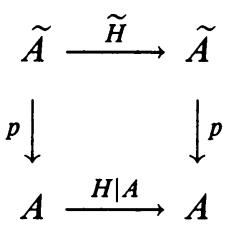

commutes, will be called a lifting of $H \mid A$. (For elements of the theory of covering spaces see for instance [8].)

Let $B$ be a two-point compactification of $\tilde{A}$. Denote the added points by $b_{0}$ and $b_{1}$. Hence $B=\tilde{A} \cup\left\{b_{0}, b_{1}\right\}$. Notice that $B$ is homeomorphic to a disk and we may assume that $B$ is a subset of $R^{2}$.

Lemma P1. The set $p^{-1}(X) \cup\left\{b_{0}, b_{1}\right\}$ is a continuum.

Proof. We can write $X=\bigcap_{n=1}^{\infty} A_{n}$, where each $A_{n}$ is a piecewise linear disk with holes, $A_{1}=A$, and $A_{n-1} \supset A_{n}$ for $n=1,2, \ldots$. Since $X$ separates $A$ between $C_{1}$ and $C_{2}$, so does each $A_{n}$. Hence, for each $n=1,2, \ldots$, the set $p^{-1}\left(A_{n}\right) \cup\left\{b_{0}, b_{1}\right\}$ is a continuum. Therefore, the set $p^{-1}(X) \cup\left\{b_{0}, b_{1}\right\}$, which is the intersection of the sets $p^{-1}\left(A_{n}\right) \cup\left\{b_{0}, b_{1}\right\}$, is a continuum.

Lemma P2. If $X$ has exactly two complementary domains, then the continuum $p^{-1}(X) \cup\left\{b_{0}, b_{1}\right\}$ does not separate the plane.

Proof. We may assume that each of the sets $A_{n}$ defined in the proof of Lemma $\mathrm{P} 1$, is an annulus. In this case, each of the sets $p^{-1}\left(A_{n}\right) \cup\left\{b_{0}, b_{1}\right\}$ is a disk, and $Y$ is the intersection of a nested sequence of disks. Hence $Y$ is a nonseparating plane continuum.

Lemma P3. If $x_{0}$ is a fixed point of $H$ in $A$, then there is a lifting of $H \mid A$, $\widetilde{H}_{1}: \widetilde{A} \rightarrow \widetilde{A}$, having exactly one fixed point in $p^{-1}\left(x_{0}\right)$, and there is a lifting of $H \mid A, \widetilde{H}_{2}: \widetilde{A} \rightarrow \tilde{A}$, without a fixed point in $p^{-1}\left(x_{0}\right)$. 
Proof. Let $\widetilde{H}$ be a lifting of $H \mid A$. Since $H$ is orientation reversing on $A$ and $H\left(C_{i}\right)=C_{i}$ for $i=1,2, \widetilde{H}$ is orientation reversing on $\widetilde{A}$ and $\widetilde{H}\left(p^{-1}\left(C_{i}\right)\right)=$ $p^{-1}\left(C_{i}\right)$ for $i=1,2$. Let $Z$ be the set of integers. There is an integer $n_{0}$, and there is a one-to-one and onto function $\mu: p^{-1}\left(x_{0}\right) \rightarrow Z$ such that $\mu \circ \widetilde{H} \circ \mu^{-1}(n)=-n+n_{0}$ for every $n \in Z$. Notice that $\widetilde{H}$ has a fixed point $\mu^{-1}\left(n_{0} / 2\right) \in p^{-1}\left(x_{0}\right)$ iff $n_{0}$ is even. If $\widetilde{H}$ has a fixed point in $p^{-1}\left(x_{0}\right)$, let $\widetilde{H}_{1}=\widetilde{H}$, and let $\widetilde{H}_{2}$ be such that $\widetilde{H}_{2}(x)=\mu^{-1}(\mu(\widetilde{H}(x))+1)$ for $x \in p^{-1}\left(x_{0}\right)$. Then $\mu \circ \widetilde{H}_{2} \circ \mu^{-1}(n)=-n+n_{0}+1$, and $\widetilde{H}_{2}$ has no fixed points in $p^{-1}(X)$. If $\widetilde{H}$ has no fixed points in $p^{-1}\left(x_{0}\right)$, let $\widetilde{H}_{2}=\widetilde{H}$ and let $\widetilde{H}_{1}$ be such that $\widetilde{H}_{1}(x)=\mu^{-1}((\mu \tilde{H}(x))+1)$ for $x \in p^{-1}\left(x_{0}\right)$.

Lemma P4. If $\widetilde{H}$ is a lifting of $H \mid A$, then $\widetilde{H}$ can be extended to a homeomorphism of the plane $R^{2}$ onto itself.

Proof. Let $K$ be a properly imbedded arc in $A$ intersecting each of the simple closed curves $C_{i}$ at exactly one point. For $n=0, \pm 1, \pm 2, \ldots$, denote by $\widetilde{A}_{n}$ the closure of a component of $p^{-1}(A-K)$ in such a way that $\tilde{A}_{n} \cap \tilde{A}_{n+1} \neq \varnothing$. For each $n$, the union $\bigcup_{k=-n}^{n} \widetilde{A}_{k}\left[\bigcup_{k=-n}^{n} \widetilde{H}\left(\tilde{A}_{k}\right)\right]$ separates $B$ between $b_{0}$ and $b_{1}$. For each $n>0$, there is an $m$ such that $\bigcup_{k=-\infty}^{-n} \tilde{A_{k}} \supset \bigcup_{k=m}^{\infty} \tilde{H}\left(\tilde{A_{k}}\right)$ and $\bigcup_{k=n}^{\infty} \tilde{A}_{k} \supset \bigcup_{k=-\infty}^{-m} \tilde{H}\left(\tilde{A}_{k}\right)$. Therefore, if $\left\{p_{n}\right\}$ is a sequence of points in $\tilde{A}$ such that $\lim _{n \rightarrow \infty} p_{n}=b_{0}\left[\lim _{n \rightarrow \infty} p_{n}=b_{1}\right]$, then $\lim _{n \rightarrow \infty} \widetilde{H}\left(p_{n}\right)=$ $b_{1}\left[\lim _{n \rightarrow \infty} \widetilde{H}\left(p_{n}\right)=b_{0}\right]$. This implies that $\widetilde{H}$ can be extended first to a homeomorphism of $B$ onto itself, and then to a homeomorphism of $R^{2}$ onto itself.

The proof of the following lemma is similar to the proof of Lemma P3 and it will be omitted.

Lemma P5. Let $U \subset A$ be a complementary domain of $X$ invariant under $H$. Then there is a lifting $\widetilde{H}$ of $H \mid A$ such that $\widetilde{H}(V)=V$ for some component $V$ of $p^{-1}(U)$.

\section{TWO COMPLEMENTARY DOMAINS}

Lemma 1. If $X$ has exactly two complementary domains, then $h$ has at least two fixed points in $X$.

Proof. Let $U$ be the bounded complementary domain of $X$. There exists a closed disk $D_{1}$ in $U$ and a homeomorphism $g_{1}$ of $R^{2}$ onto itself such that $g_{1} \circ h\left(D_{1}\right)=D_{1}$ and $g_{1}(x)=x$ outside $U$. Similarly, assuming that the unbounded complementary domain of $X$ is a neighborhood of the added point of a one-point compactification of $R^{2}$, we can construct a homeornorphism $g_{2}$ of the plane such that $g_{2}(x)=x$ for $x \in X \cup U$, and $g_{2} \circ h\left(D_{2}\right)=D_{2}$ for some closed disk $D_{2}$ containing $X$. Let $H=g_{2} \circ g_{1} \circ h$. Notice that $H|X=h| X$, $H$ is an orientation reversing homeomorphism of the plane, and there exists an 
annulus $A$, containing $X$, which is invariant under $H$, and whos eboundary components are in different complementary domains of $X$.

Let $\widetilde{H}$ be a lifting of $H \mid A$ as defined in $\S 2$.

We will show that $\widetilde{H}$ has a fixed point in $p^{-1}(X)$. As in the previous section, let $B$ be a two-point compactification of $\tilde{A}$, and let the added points be denoted by $b_{0}$ and $b_{1}$. By Lemma P2, the set $Y=p^{-1}(X) \cup\left\{b_{0}, b_{1}\right\}$ is a nonseparating plane continuum. Let $f$ be a homeomorphism of $R^{2}$ onto itself such that $f \mid p^{-1}(X)=\widetilde{H}$ (see Lemma P4). By Bell's theorem, $f$ has a fixed point in $Y$. Since $f\left(b_{0}\right)=b_{1}$ and $f\left(b_{1}\right)=b_{0}, \widetilde{H}$ has a fixed point in $p^{-1}(X)$.

First, we will show that $H$ (equivalently $h$ ) has at least one fixed point in $X$. If $H$ has no fixed points in $X$, then $\widetilde{H}$ has no invariant fibers $p^{-1}(x)$ with $x \in X$. Hence $\widetilde{H}$ has no fixed points in $p^{-1}(X)$, which contradicts the above.

Next, we will show that $H$ has at least two fixed points in $X$. Suppose to the contrary that $x_{0} \in X$ is the only fixed point of $H$ in $X$. The only possible fixed point of $\widetilde{H} \mid p^{-1}(X)$ is in $p^{-1}\left(x_{0}\right)$. By Lemma $\mathrm{P} 3$, there is a lifting $\widetilde{H}_{2}$ of $H \mid A$ without a fixed point in $p^{-1}\left(x_{0}\right)$ and hence without a fixed point in $p^{-1}(X)$. By Lemma P4, $\widetilde{H}_{2}$ can be extended to a homeomorphism $R^{2}$ without fixed points in $Y$ which, again, contradicts Bell's theorem.

\section{The genERAL CASE}

Denote by:

(1) $[X, h]$ the union of $X$ and the bounded complementary domains of $X$ which contain no fixed points of $h$,

(2) $P(X, h)$ the set of fixed points of $h$ in the bounded complementary domains of $X$,

(3) $L P(X, h)$ the set of the limit points of $P(X, h)$ in $X$,

(4) $Q(X, h)$ the set of the fixed points of $h$ in $X$.

Proposition 1. The continuum $[X, h]$ is invariant under $h$.

Proposition 2. $Q(X, h)=Q([X, h], h)$.

Proposition 3. If $L P(X, h)=\varnothing$, then $[X, h]$ has finitely many complementary domains.

Proposition 4. $L P(X, h) \subset Q(X, h)$.

The following lemma is an immediate consequence of Bell's theorem.

Lemma 2. If $[X, h]$ does not separate $R^{2}$, then $h$ has a fixed point in $X$.

The proofs of Lemmas 3 and 4 are based on a construction similar to that of the proof of Lemma 1. 
Lemma 3. If $\operatorname{LP}(X, h)$ consists of exactly one point, then there is an orientation reversing homeomorphism $f$ of $R^{2}$ onto itself, and there exists a continuum $Y$ invariant under $f$ such that (1) $Y$ has finitely many complementary domains, and (2) if $Q(X, h)$ contains $n$ (finitely many) points, then $Q(Y, f)$ contains at most $n-1$ points.

Proof. Let $U_{1}$ be a bounded complementary domain of $X$ invariant under $h$. Denote by $U_{2}$ the unbounded complementary domain of $X$. Let $A$ be an annulus in $R^{2}$ with boundary components $C_{1}$ and $C_{2}$ contained in $U_{1}$ and $U_{2}$ respectively. There is an orientation reversing homeomorphism $H$ of $R^{2}$ such that $A$ is invariant under $H$, and $H(x)=h(x)$ for $x$ in an open neighborhood of $k^{2}-\left(U_{1} \cup U_{2}\right)$. Then $L P(X, h)=L P(X, H)$.

Denote by $x_{0}$ the only point of $L P(X, h)$.

Let $\tilde{A}$ be the universal covering space of $A$, and let $p: \widetilde{A} \rightarrow A$ be the covering map. Let $\widetilde{H}: \widetilde{A} \rightarrow \widetilde{A}$ be a lifting of $H \mid A$ with no fixed points in the fiber $p^{-1}\left(x_{0}\right)$ (see Lemma P4).

Let $B=\tilde{A} \cup\left\{b_{0}, b_{1}\right\}$ be a two-point compactification of $\tilde{A}$, embedded in $R^{2}$, and $f$ a homeomorphism of the plane such that $f \mid \widetilde{A}=\widetilde{H}$ ( $f$ interchanges the points $b_{0}$ and $\left.b_{1}\right)$. By Lemma $\mathrm{P} 1$ the set $p^{-1}(X) \cup\left\{b_{0}, b_{1}\right\}$ is a continuum. Let $Y=\left[p^{-1}(X) \cup\left\{b_{0}, b_{1}\right\}, f\right]$. By Proposition $1, Y$ is invariant under $f$, and by Proposition 2, $Q(Y, f)=Q\left(p^{-1}(X) \cup\left\{b_{0}, b_{1}\right\}, f\right)$. If $y$ is a fixed point of $f$ in $\tilde{A}$, then $y \in p^{-1}(x)$, where $x$ is a fixed point of $H$ in $A$. Hence $L P(X, H) \cap p(Q(Y, f)) \supset p(L P(Y, f))$ (see Proposition 4) and therefore $L P(Y, f)=\varnothing$. By Proposition 3, $Y$ has finitely many complementary domains. Since for each $x \in X$, the set $p^{-1}(x)$ contains at most one fixed point of $\widetilde{H}$, and $\widetilde{H}$ has no fixed points in $p^{-1}\left(x_{0}\right)$, the cardinality of $Q(Y, f)$ is not greater than the cardinality of $Q(X, h)$ minus 1 .

Lemma 4. Let $k>2$ be an integer. If $[X, h]$ has $k$ complementary domains, then there exist an orientation reversing homeomorphism $f$ of $R^{2}$ onto itself, a continuum $Y$ invariant under $f$, and an integer $j, 2 \leq j \leq k-1$, such that (1) $Y$ has $j$ invariant complementary domains, and (2) the cardinality of $Q(Y, f)$ does not exceed the cardinality of $Q(X, h)$.

Proof. Let $U_{1}, \ldots, U_{k-1}$ be the bounded complementary domains of $[X, h]$, and let $U_{k}$ be the unbounded complementary domain.

There is an annulus $A$ in $R^{2}$ with boundary components $C_{1}$ and $C_{2}$ in $U_{k-1}$ and $U_{k}$ respectively, and there is an orientation reversing homeomorphism $H$ of the plane such that $H(x)=h(x)$ for $x \in X$ and $H(A)=A$.

Let $\tilde{A}$ be the universal covering space of $A, p: \widetilde{A} \rightarrow A$ the covering map, and let $\widetilde{H}: \widetilde{A} \rightarrow \widetilde{A}$ be a lifting of $H \mid A$ such that there is a component $V_{1}$ of $p^{-1}\left(U_{1}\right)$ which is invariant under $\widetilde{H}$ (see Lemma P5). Let $B=\tilde{A} \cup\left\{b_{0}, b_{1}\right\}$ be a two-point compactification of $\tilde{A}$, embedded in the plane and let $f$ be a homeomorphism of the plane such that $f \mid \widetilde{A}=\widetilde{H}$. By Lemma Pi, the set 
$\widehat{Y}=p^{-1}([X, h]) \cup\left\{b_{0}, b_{1}\right\}$ is a continuum. Let $Y=[\widehat{Y}, f]-V_{1}$. Notice that $Y$ is a continuum invariant under $f$ with at least two invariant complementary domains.

For each $1 \leq i \leq k-2$, there is at most one component of $p^{-1}\left(U_{i}\right)$ which is invariant under $\widetilde{H}$. Therefore, the number $j$ of the complementary domains of $Y$ satisfies the inequality $2 \leq j \leq k-1$.

The only fixed points of $f$ in $Y$ are in the fibers $p^{-1}(x)$, where $x \in X$ is a fixed point of $h$. Since there is at most one fixed point of $f$ in such a fiber, the cardinality of $Q(Y, f)$ does not exceed the cardinality of $Q(X, h)$.

Theorem. If at least one of the bounded complementary domains of $X$ is invariant under $h$, then $h$ has at least two fixed points in $X$. Otherwise $h$ has at least one fixed point in $X$.

Proof. By Lemma 2, if there are no invariant bounded complementary domains, then $h$ has a fixed point in $X$.

If $L P(X, h)$ contains more than one point, then clearly $h$ has at least two fixed points in $X$.

By Lemma 3 , if $L P(X, h)$ contains exactly one point which is the only point of $Q(X, h)$, then there exists an orientation reversing homeomorphism $f$ of the plane and a continuum $Y$ invariant under $f$, such that $f$ has no fixed points in $Y$, and $Y$ has finitely many complementary domains. If $[Y, f]$ does not separate the plane, then $f$ has a fixed point in $Y$. If $[Y, f]$ separates the plane, then by Lemma 4 applied inductively, $f$ has a fixed point in $Y$. Hence, $Q(X, h)$ contains at least two points.

Assume now that $X$ has at least one invariant bounded complementary domain $U$. If $L P(X, h)=\varnothing$, then $[X, h]$ has finitely many complementary domains. The continuum $[X, h] \cup U$ separates the plane. Either $[X, h] \cup U$ has exactly two complementary domains, or by Lemma 4, applied inductively, we obtain an orientation reversing homeomorphism $g$ of the plane having an invariant continuum $Z$ with exactly two complementary domains. By Lemma $1, g$ has at least two fixed points in $Z$. Therefore, $h$ has at least two fixed points in $X$.

Remark. The following question, asked W. Kuperberg, seems to be related to the above theorems:

Let $A$ be an annulus, and let $\varphi$ be an orientation reversing homeomorphism of the annulus $A$ onto itself which does not interchange the two boundary components of the annulus. Do there always exist two disjoint subcontinua $F_{1}$ and $F_{2}$ of the annulus, each invariant under $\varphi$, such that the set $F_{1} \cup F_{2}$ separates the annulus?

In response to this question, $\mathrm{H}$. Bell [3] constructed two interesting examples of orientation reversing homeomorphisms, satisfying the above conditions, and for which such continua do not exist. 
Let $A$ be the annulus defined in polar coordinates by $A=\{(r, \theta): 1 \leq r \leq$ 2\}. Bell proved that:

(i) There is an orientation reversing homeomorphism $h_{1}: A \rightarrow A$ such that

(1) if $r=1$ or $r=2$, then $h_{1}(r, \theta)=(r,-\theta)$,

(2) if $1<r<2$, then the closure of $\left\{h_{1}^{i}(r, \theta): i=1,2, \ldots\right\}$ contains the set $\{(r, \theta): r=1\}$,

(3) if $1<r<2$, then the closure of $\left\{h_{1}^{-i}(r, \theta): i=1,2, \ldots\right\}$ contains the set $\{(r, \theta): r=2\}$.

(ii) There is an orientation reversing homeomorphism $h_{2}: A \rightarrow A$ such that

(1) if $r=1$ or $r=2$, then $h_{2}(r, \theta)=(r,-\theta)$,

(2) if $1<r<2$, then $\lim _{i \rightarrow \infty} h_{2}^{i}(r, \theta)=(1,0)$,

(3) if $1<r<2$, then $\lim _{i \rightarrow \infty} h_{2}^{-i}(r, \theta)=(2,0)$.

\section{REFERENCES}

1. Harold Bell, $A$ fixed point theorem for planar homeomorphisms, Bull. Amer. Math. Soc. 82 (1976), 778-780.

2. __ A fixed point theorem for plane homeomorphisms, Fund. Math. 100 (1978), 119-128.

3.

4. Beverly Brechner, Prime ends, indecomposable continua, and the fixed point property; Topology Proc. 4 (1979), 227-234.

5. Morton Brown, A short short proof of the Cartwright-Littlewood fixed point theorem, Proc. Amer. Math. Soc. 65 (1977), 372.

6. M. L. Cartwright and J. C. Littlewood, Some fixed point theorems, Ann. of Math. 54 (1951), 1-37.

7. O. H. Hamilton, A short proof of the Cartwright-Littlewood fixed point theorem, Canad. J. Math. 6 (1954), 522-524.

8. Sze-Tsen Hu, Homotopy theory, Academic Press, 1959.

Department of Foundations, ANalysis and Topology, Auburn University, Auburn, Alabama 36849-5310 